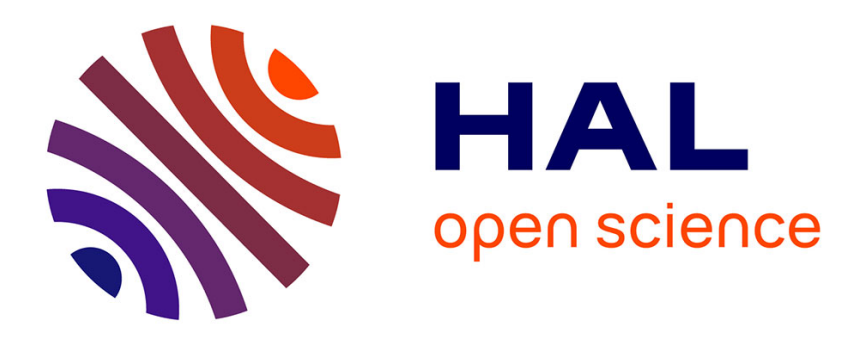

\title{
BOISIC : l'information télématique pour les entreprises du secteur bois
}

Pierre Guillon

\section{To cite this version:}

Pierre Guillon. BOISIC : l'information télématique pour les entreprises du secteur bois. Revue forestière française, 1993, 45 (S), pp.63-67. 10.4267/2042/26502 . hal-03443952

\section{HAL Id: hal-03443952 \\ https://hal.science/hal-03443952}

Submitted on 23 Nov 2021

HAL is a multi-disciplinary open access archive for the deposit and dissemination of scientific research documents, whether they are published or not. The documents may come from teaching and research institutions in France or abroad, or from public or private research centers.
L'archive ouverte pluridisciplinaire HAL, est destinée au dépôt et à la diffusion de documents scientifiques de niveau recherche, publiés ou non, émanant des établissements d'enseignement et de recherche français ou étrangers, des laboratoires publics ou privés. 


\title{
BOISIC : L'INFORMATION TÉLÉMATIQUE POUR LES ENTREPRISES DU SECTEUR BOIS
}

\author{
P. GUILLON
}

La recherche d'une meilleure information économique et commerciale des entreprises du secteur bois a progressivement conduit à élaborer le système BOISIC. Son service télématique (1) constitue un outil de gestion au service des entreprises, en même temps que le lien interrégional entre différents organismes chargés du développement de l'économie du bois.

\section{INFORMATION POUR LES PROFESSIONNELS DU BOIS : LES CARENCES ACTUELLES}

Les besoins d'information des professionnels, et plus particulièrement des responsables d'entreprises, dans le domaine économique et commercial, consistent surtout en quatre types de données.

Opportunités d'achat ou vente, petites annonces

La source la plus riche est incontestablement I'hebdomadaire Le Bois national, avec l'avantage de la fréquence et de la variété. Mais ses annonces ne sont pas classées par localisation, ni avec assez de détail par catégories.

D'autres annonces sont publiées dans des supports très divers (bulletins régionaux d'affaires, le Moniteur du Commerce international, etc.).

Fichiers de clients ou fournisseurs potentiels

Les nombreux fichiers d'entreprises disponibles présentent de grosses lacunes pour un usage commercial: ils sont le plus souvent imprécis ou incomplets, ne détaillent pas la production et n'indiquent généralement rien des achats, du type d'équipement, de savoir-faire, ni du type de clientèle.

\section{Statistiques concernant marchés et ressources}

Elles sont très diverses et rarement rassemblées à l'échelle régionale ou départementale.

Adresses et services offerts par les organismes professionnels ou administratifs

Là aussi on note un manque de sélection ou de clarté de présentation.

(1) Accès Minitel par 3616 code BoIsıC. 
Quant au moyen de diffusion, le papier, auquel on est habitué, il permet une lecture au rythme voulu et une réflexion active; mais il présente l'inconvénient de sa distribution longue et onéreuse et parfois du long délai d'accès à l'information très précise que l'on recherche.

Le problème s'est donc posé de réaliser un système d'information permettant de compléter les moyens existants et répondant mieux aux besoins décelés, tant par la nature des données proposées que par les supports et le mode de consultation.

\section{L'ÉLABORATION DE BOISIC}

Plusieurs étapes jalonnent la mise au point progressive de BOISIC au cours des années 80 :

- L'inventaire des industries du bois et du meuble dans les régions Limousin et PoitouCharentes ${ }^{(2)}$, au cours de l'été 1979, permet de constituer un fichier pratiquement exhaustif indiquant pour chaque entreprise :

- renseignements généraux ;

- achats, produits et relations de sous-traitance comme donneur d'ordres ou preneur d'ordres, selon une nomenclature à plus de 500 catégories (cf. annexe, p. 67);

- types d'équipement ou savoir-faire;

- types de clientèle.

Ce fichier, mis à jour chaque année, fut diffusé sous forme de catalogue (1980 et 1984), puis de jeux de fiches sur demande, puis par Minitel (1987).

C'est la partie la plus riche du système, avec environ 1000 entreprises actuellement présentées.

- La création de la Bourse des Bois du Centre-Ouest, en 1980, donne lieu à l'édition d'un bulletin d'annonces d'abord mensuel, puis bimensuel en raison du besoin d'une mise à jour plus fréquente.

Après mise en accès télématique en 1986, la fréquence du bulletin-papier est réduite à 8 par an.

Suite aux chablis de Bretagne en 1987, les annonces de travaux forestiers et de coupes de bois sur pied ont été ajoutées à celles qui préexistaient.

À partir de 1989, s'y adjoignent les annonces de la Bourse des Bois de Bourgogne.

Puis, en 1990, celles des Bourses des Bois de Bretagne et de Rhône-Alpes.

BOISIC offre ainsi 150 à 200 annonces, qui se renouvellent régulièrement.

- Le fichier des scieries membres de la Bourse des Bois, indiquant en détail la production et le stock de chacune par catégorie de bois scié, est mis à jour chaque année depuis 1983. II est diffusé sur papier depuis lors et par Minitel depuis 1987.

- Le fichier des entreprises forestières (pépinières, entreprises de service, fournisseurs de matériel forestier, etc.) a été ajouté en 1990, à la demande du Centre régional de la Propriété forestière de Normandie. II indique pour chacune, outre les renseignements généraux, ses activités (une trentaine de catégories) et sa zone d'action.

- Enfin, diverses informations, ainsi que les adresses d'organismes relatifs au secteur bois sont diffusées dans deux chapitres du service télématique, ou par le biais de notes d'informations

(2) En raison du poids des activitès liées au bois dans ces deux régions (plus de $15 \%$ des salariés de l'industrie), la Chambre régionale de Commerce et d'Industrie (CRCI) avait créé une "mission bois " dès 1978. 
propres à chacune des régions, en même temps qu'une revue de presse régionale, des comptes rendus de réunions, etc.

- Ce développement du champ d'informations s'est accompagné de l'extension du champ géographique couvert par BOISIC : successivement Limousin et Poitou-Charentes, puis Bourgogne, Bretagne, Haute et Basse-Normandie, et Rhône-Alpes.

Cette collaboration interrégionale se base sur des conventions bilatérales.

Le réseau BOISIC réunit ainsi les organismes suivants :

- AIFOBAN

35, rue des Grandes-Poteries - 61000 ALENÇON

Tél. 33.32.14.19 - Fax. 33.32.94.53

- ANORIBOIS

24, quai Gaston-Boulet - 76000 ROUEN

Tél. 35.15.49.99 - Fax. 35.15.01.59

- APROVALBOIS

16 bis, boulevard des Brosses - 21000 DIJON

Té'. 80.44.33.78 - Fax. 80.44.33.43

- COCEB-BOIS

2, cours des Alliés - 35029 RENNES CEDEX

Tél. 99.30.88.89 - Fax. 99.30.89.08

- CRCI Limousin-Poitou-Charentes

Boulevard des Arcades - 87038 LIMOGES CEDEX

Tél. 55.04.40.22 - Fax. 55.04.40.40

- CRPF Normandie

15, rue de Vaucelles - BP 2006 - 14019 CAEN CEDEX

Tél. 31.82.21.14 - Fax. 31.78.76.31

- FIBRA

34, rue Casimir-Périer - 69002 LYON

Tél. 78.37.09.66 - Fax. 78.37.83.62

- La réalisation de BOISIC a nécessité un investissement en conception, en logiciel et en lancement publicitaire partagé entre les sept régions avec le soutien financier de la Direction de l'Espace rural et de la Forêt ainsi que des collectivités locales (Conseils régionaux et généraux).

La réalisation du logiciel et l'hébergement du service ont été confiés en 1986 à la Chambre de Commerce et d'Industrie de Montpellier, qui possédait une expérience comparable dans le domaine de la sous-traitance sur plusieurs régions.

Les coûts de fonctionnement (mise à jour des données, serveur) sont partagés entre les organismes associès.

\section{AUTRES SERVICES TÉLÉMATIQUES POUR LE COMMERCE DU BOIS}

Après la création de BOISIC, d'autres services télématiques concernant le commerce du bois sont apparus:

3617 TELBOIS (Bois-Contact à Raddon, Haute-Saône),

3616 MEDIABOIS (à Paris),

3616 BOIS (Fédération française du Commerce du Bois, à Paris), 


\section{P. GUILLON}

3617 TBOIS (Le Bouscat, Gironde),

3614 CANALBOIS (Le Journal du Bois, à Paris),

3616 EB (Eurobois à Dijon, Côte-d'Or),

3616 XYLVA (Montpellier, Hèrault), ...

Tous ces services présentent des annonces commerciales d'offre ou de demande de grumes ou de sciages, et parfois des listes d'entreprises, mais sans indications d'ordre technique ni économique. Certains semblent en sommeil depuis plusieurs années, voire arrêtés. Les services d'annonces les plus fournis sont proposés par EB (environ 150 annonces, essentiellement du Nord-Est), et par TBOIS (surtout des ventes de lots de bois sur pied par experts forestiers).

Enfin, d'autres services diffusent des informations relatives au bois, mais dans d'autres perspectives :

3616 CTBA : Présentation d'activité et rèsultats de recherche,

3614 INFOBOIS: Communication des CRPF aux propriétaires forestiers,

3615 ONF : Information sur les ventes de bois en forêts soumises,

3614 UNICOF: Information sur les ventes de bois par les coopératives, ainsi que quelques autres émanant de sociétés commerciales privées.

\section{BOISIC : UN SYSTĖME D'INFORMATION EN ÉVOLUTION}

Pour répondre au besoin des entreprises du secteur bois, le système BOISIC a èvolué en nature d'informations proposées, en présentation de ces informations, et a étendu son champ géographique.

D'autres développements sont en préparation dans chacun de ces trois domaines, avec l'objectif d'offrir aux professionnels du bois, à l'échelle interrégionale, une meilleure information commerciale, laquelle est un facteur essentiel de leur succès.

P. GUILLON

CHAMBRE RÉGIONALE DE COMMERCE ET D'INDUSTRIE LIMOUSIN-POITOU-CHARENTES

Boulevard des Arcades 87038 LIMOGES CEDEX 


\section{BOISIC : \\ Codes produits et activités au 15/07/1993}

OOOO FORÊT : PRODUITS ET SERVICES

0010 Organismes forestiers

0100 Plants et graines

0110 Plants de peupliers

0120 Plants feuillus

0130 Plants résineux

0140 Graines torestières

0200 Fournitures forestières

D300 Matériel torestier

0400 Travaux torestiers

0500 Conseil et gestion forestière

$\ldots$

0600 Exploitation forestière

0700 Bois sur pied

$\ldots$

1000 BOIS ROND ET SOUS-PRODUITS

1100 Grumes feuillus tempérés

1110 Grumes chêne

1111 Grumes chêne tranchage

1112 Grumes chêne sciage

1120 Grumes peuplier

1200 Grumes teuillus Amérique

1211 Grumes Chêne Amérique

1212 Grumes Merisier Amérique

1213 Grumes Noyer Amérique

1214 Grumes Érable Amérique

1300 Grumes tropicales
1400 Grumes de résineux

$\cdots$

1500 Rondins trituration feuillus

$\ldots$

1600 Rondins trituration résineux

1700 Bois d'industrie

1800 Sous-produits bois

1900 Bois de feu

...

2000 BOIS SCIÉ

3000 MATÉRIAUX À BASE DE BOIS

...

4000 PRODUITS/BOIS POUR CONSTRUCTION

$\cdots$

5000 EMBALLAGES EN BOIS

$\ldots$

6000 COMPOSANTS/BOIS POUR MEUBLES

...

7000 MEUBLES

...

8000 ARTICLES DE LOISIR OU MÉNAGERS

9000 DPÉRATIONS

* Seuls les postes des deux premières rubriques ont été détaillés. L'annexe complète peut être oblenue sur simple demande auprès du Secretariat de Rédaction de la Revue forestière française. 